\title{
"It's Just a Lot Different Being Male Than Female in the Sport": An Exploration of the Gendered Culture Around Body Pressures in Competitive Figure Skating
}

\author{
Dana K. Voelker \\ West Virginia University \\ Justine J. Reel \\ University of North Carolina Wilmington
}

\begin{abstract}
Figure skaters experience pressure associated with their sport to change their body weight, shape, or size to meet appearance and performance expectations. Figure skaters may experience different body-related expectations based on gender despite performing in identical or similar training and competition environments. In a qualitative investigation that examined body pressure experiences of male skaters, participants discussed some of their struggles, but seemed compelled to discuss, unexpectedly, the plight of female skaters in facing the skating body ideal. The present findings represent an exploratory analysis of qualitative data elucidating the body pressure experiences of female skaters through the eyes of male skaters. Participants were 13 competitive male figure skaters ages 16-24 $(M=18.53)$. Analyzed using a social constructivist and critical perspective, the results demonstrated the salience of body pressures for female skaters and afforded insight into sociocultural and historical factors that influence how male and female skaters experience their bodies differently in a skating context. Male skaters reported they faced less extreme body pressures, had certain physical advantages, and tended to be more confident than female skaters, which underscored a gendered body pressure experience. This work explores the intersections of gender and power within figure skating and examines body image concerns and unhealthy eating and exercise behaviors as a larger social justice issue that serves to encourage similar investigations in other sports.
\end{abstract}

Keywords: body image, eating disorders, female athlete, sociocultural perspectives

Figure skaters are evaluated on the execution of challenging technical skills as well as how those skills are packaged with other performance components, including costume, music, choreography, and physical appearance (Cummins, 2007). Male and female skaters report experiencing pressure from a figure skating culture that socializes them to desire changing their body weight, shape, size, or appearance to meet performance expectations. In a qualitative interview study, female competitive skaters described immense pressure to meet narrow, often unattainable, and inflexible body ideals. These female skaters were subjected to bodyshaming comments from coaches, parents, and skating partners that contributed to self-consciousness about appearance and, in some cases, led skaters to use weight management strategies that compromised health and performance (Voelker \& Reel, 2015). In a separate study, male competitive skaters identified the need to build muscle without too much bulk and stay lean for performance; however, the interviewees reported these body ideals were often flexible and positive. Further, the male skaters expressed general confidence in their bodies both in terms of function and appearance (Voelker \& Reel, 2018). In figure skating settings, male and female skaters share the same practice sessions and tend to interact with

Voelker is with the College of Physical Activity \& Sport Sciences, West Virginia University, Morgantown, WV. Reel is with the College of Health \& Human Services, University of North Carolina Wilmington, Wilmington, NC. Voelker (dkvoelker@mail.wvu.edu) is corresponding author. one another in rink lobbies and at competitions. However, these qualitative findings suggest men and women experience their skating environments, and their bodies, differently. Within a skating context, gender therefore serves as a vital lens through which to understand these phenomena.

The gender binary and traditional body ideals have been strongly upheld in contemporary figure skating; female skaters are rewarded for qualities such as grace, beauty, and weightlessness, while male skaters are applauded for demonstrations of strength, power, and force (Adams, 2011). Lee (1994) contended the "body is a text of culture; it is a symbolic form upon which the norms and practices of a society are inscribed" (pp. 333-334). Accordingly, critical examination of figure skating's history reveals how gender has been constructed specifically in this context, including how masculinity and femininity have been defined and then reflected in the expectations for male and female skaters' bodies. In her detailed analysis, Adams (2007) described the oft forgotten male-dominated origins of skating in 18th century England. Masculinity in skating was defined by a "careful mix of feeling and control" (p. 875) and reflected the masculine ideals of the privileged elite (e.g., expressive but refined). Various historical accounts point to men using skating to express beauty and grace rather than exhibit strength, speed, height, or "bravado" (p. 878). Among the postulations on why women rarely participated, Adams explained skating violated traditional definitions of femininity. Skating, a physical activity, could have been thought to pose health risks, be incompatible with typical women's clothing, or 
represent an unprecedented, and unwanted, form of women's emancipation (Adams, 2007). Male, but not female, bodies served a socially and culturally prescribed purpose in skating, and power and privilege in skating during this era appeared to rest with men.

In the 19th century, skating evolved into a mixed-gender sport as more women participated and, in the 1920s through 1940s, public interest in women's skating grew-a phenomenon largely attributed to Sonja Henie (Adams, 2010). A three-time Olympic Champion, 10-time World Champion, and movie star, Henie is known for popularizing the artistic and aesthetic features of skating as evidenced in, for example, the introduction of white skating boots and shorter skirts. Henie has been identified as a pivotal influence on the hyperfeminization of figure skating that was, and continues to be, perpetuated in modern-day media (Adams, 2010). Fabos (2001) and Feder (1994) offered detailed accounts of mediaconstructed narratives that contextualize female skaters as princesses in a fairytale - dependent, vulnerable, and sexually available objects of beauty epitomized as artists versus athletes-to align with mainstream gender ideology. Fabos (2001) explained female skaters who violate these ideals are villainized regardless of their skating quality. Through its manipulated story-telling, the media, then, has played a critical role in branding figure skating as a girl's sport most suitable for ladies.

Figure skating's historical shift from a manly to hyperfeminized activity poses interesting questions relative to intersections of gender and power. Several scholars point to the marginalization of women and girls in figure skating despite representing a majority of participants. Adams (2011), for example, described how rules outlined by the International Skating Union systematically allow male skaters to achieve higher scores than female skaters based, in part, on the opportunity for longer programs. Story and Markula (2017) discussed age requirements that systematically permit male skaters to compete at a given level longer, providing them more time to actualize their potential and extend their careers. Other evidence suggests gendered cultural narratives from coaches, that promote aggression in male skaters and passivity in female skaters, may serve to disempower female skaters and compromise their ability to excel technically (McGarry, 2005). Based on the existing sociocultural and historical literature on figure skating, critical examination of dominant gender discourse and its impact on skaters' lived experiences is vital to shaping positive, safe, and equitable skating environments, especially with respect to their bodies.

The present findings, not published previously, are the result of an exploratory analysis of qualitative data collected by Voelker and Reel (2018) to address a new research question that elucidates how female skaters are affected by body pressures, also referred to as weight pressures, through the eyes of male skaters. Voelker and Reel interviewed male skaters to examine their body pressure experiences in figure skating. While acknowledging body pressures exist for male skaters (see Voelker \& Reel, 2018), these participants shed light, unexpectedly, on the plight of female skaters in facing the skating body ideal. Through a social constructivist lens, we examined broad contextual influences of the figure skating environment to examine how body pressures are constructed and reinforced. Using Denzin's (2017) critical paradigm, we further examined gender-based power and privilege structures that shape body pressure experiences in figure skating. Foucault (1988) contended power is always present and functions by operating through us. The voices of male skaters, which represent a vehicle through which power structures operate, afforded a unique opportunity to examine how body pressure experiences of female skaters are shaped by larger sociocultural beliefs, values, and ideas, situated in the historical context of figure skating, and communicated through dominant discourse. Denzin (2017) wrote through qualitative inquiry, "we are no longer called to interpret the world ... we are called to change the world" (p. 9). Integrating social constructivist and critical paradigms allowed us to contextualize body image concerns experienced by skaters, not as psychological shortfalls, but as a reflection of broader social justice concerns. By actively critiquing the dominant discourse as told by male skaters, we could identify areas for needed change.

\section{Methods}

\section{Participants}

Participants were 13 competitive male figure skaters ages 16-24 $(M=18.53, S D=3.33)$ interviewed in Voelker and Reel (2018). Skaters were from 12 figure skating clubs in seven U.S. states and one Canadian province and reported an average skating experience of 10.38 years $(S D=4.05)$. Skaters identified as Caucasian $(n=9)$, Asian American/Pacific Islander $(n=1)$, Caucasian and Asian American/Pacific Islander $(n=1)$, Caucasian, Asian American/ Pacific Islander and Native American $(n=1)$, and Hispanic/ Latino/Mexican American $(n=1)$. Skaters reported their highest level of competition at national $(n=8)$ and international events $(n=5)$ in singles $(n=9)$ or pairs $(n=4)$ disciplines.

\section{Procedures}

Data were collected as part of Voelker and Reel's (2018) qualitative study; a summary of procedures is provided here. Following Institutional Review Board approval, convenience and snowball sampling methods were used to identify and recruit potential participants via flyers and social media outlets to professional colleagues and members of the figure skating community (e.g., club presidents, program directors, coaches). Participants completed consent and background information forms prior to participating in a 60- to 90-minute audio-recorded interview via Skype $(n=10)$ or telephone $(n=3)$. Informed by previous research (i.e., Galli \& Reel, 2009; Voelker, Petrie, Reel, \& Gould, 2018) on body image concerns in male athletes and male figure skaters specifically, the interviewer used a semi-structured guide. The first author began each interview with rapport-building, followed by broad, open-ended questions and follow-up prompts to stimulate elaboration. Throughout data collection, the first author kept a reflexive journal to elucidate and process impressions of her interactions with each participant. A \$25 retail gift card was provided to each participant following data collection (Voelker \& Reel, 2018).

\section{Data Analysis}

Interviews were transcribed verbatim and reviewed by a threeperson research team. Case profiles to feature the main ideas, meaning, and context of each interview were constructed and discussed with 11 of 13 participants who were reached for a follow-up phone call with the first author. Eight participants shared additional thoughts, ideas, and elaborations. The interpretations of the research team and participant reflections were integrated into the remaining phases of the data analysis (Smith \& McGannon, 2018) following the steps of inductive thematic analysis outlined by Braun and Clarke (2006; see also data analysis in Voelker \& Reel, 2018). 
Although not a part of our original research question (i.e., to examine competitive male skaters' experiences of body pressures in figure skating), we examined the data yielding insight into male skaters' perceptions of female skaters' body pressure experiences using a social constructivist and critical lens. Employing a critical friend approach (Tracy, 2010) at this phase allowed the research team to voice interpretations, offer critical feedback, and consider interpretative possibilities. Specifically, the first author has direct figure skating experience while the second author, who is familiar with the literature on sociocultural aspects of sport, could offer perspective on how the data might be similar to, or different from, patterns observed in other performance contexts. This range of experience and proximity to figure skating allowed each author to challenge and deepen interpretations (e.g., although normative to this skater and stated benignly, does the language used suggest a lack of understanding of eating disorders? In what ways? Why are biological differences discussed with confidence? How and why do the male skaters in this study communicate expertise on the experiences of female skaters? Who is charged as being the knower of truths and who is not? Why?). This process of challenging others' constructions of meaning was instrumental to clarifying the power and privilege structures reflected in language and voice. Through reflective discussions, codes were organized into subthemes and themes, which remained an iterative process through the write-up phase as the results were interpreted in the context of existing literature. Pseudonyms were changed to further protect confidentiality in reporting results.

\section{Results}

\section{Perceptions of the Body Ideal for Female Figure Skaters}

Male skaters were asked to describe the ideal figure skater's body. In addition to describing the ideal body for themselves (see Voelker \& Reel, 2018), they also described the ideal body for female skaters. One participant, Tom, reported the ideal body is "pretty similar for both genders" in that female skaters "need to be fairly lean as well." However, body ideals for female skaters were discussed with greater emphasis on weight and appearance, as another participant, Eli, noted: "As long as a girl stays light and doesn't hold too much body fat then yeah she'll be good." Jay likened the ideal female skater's body to a Barbie doll:

Ladies [figure skaters] I'd say it's kind of like that Barbie look most times. The Barbie look doesn't really have too much going on most times in terms of muscles. It's all just very thin at the waist, you have the space in between your legs, and you don't have the arms going ... maybe if you take out the chest, I'd say yeah ... that's kind of the way I see female skaters, but obviously I see them as really strong underneath it. I don't think Barbie could do 10 push-ups.

Other participants explained that the right fit (i.e., body type) between male and female pairs partners was instrumental to performance success. Tom emphasized the importance of a substantial weight difference: "If you're in pairs the girl obviously has to weigh a lot less than the guy. Like an 80-pound difference." Ike, another participant, similarly explained: "I think we just got to the point that no matter how hard we tried, no matter how strong I got . . . that was just too small a weight difference for the tricks we wanted to do." Ike later mentioned puberty as one reason for their difficulty as a pairs team:

I spent years with her trying to slim down and get to that perfect body type, which was only a problem when she hit puberty. Before she hit puberty, she was actually, probably perfect. But she was one of those girls that got hit really hard ....

Ian reported achieving a specific look is sometimes seen as more important than technical skill for female pairs skaters: "It's a very touchy subject for a lot of the skaters in pairs because a lot of people will break up with their [female] partners because they're too big or they grew. Regardless of their skill level." Some participants reported they would never comment on their female skating partner's body because they "don't feel comfortable," believe they "have no say," and doing so has previously caused a "firestorm." In these instances, skating partners indicated they would defer to a coach to have those conversations. Other participants stated they could make direct recommendations, such as "watch what you eat," "work out a little bit more," or "see a nutritionist."

\section{Perceptions of the Body Pressure Experiences of Female Figure Skaters}

Although male skaters shared body pressures they experienced personally (see Voelker \& Reel, 2018), their accounts of body pressures experienced by female skaters were far more specific and vivid. In fact, some reported becoming aware of body pressures in skating for the first time, not from a personal experience, but through observations of female peers, as a participant, Tod, noted: "I just saw some of the girls were very skinny." Several examples of negative body pressures for female skaters were described. For example, Ben, another participant, explained: "Her dad sends her pictures of fat princesses . . . saying that if you don't lose weight, this is what you're going to look like." Leo offered another example of body pressures from parents: "Her dad heard this rumor that if you don't get your double axel before your first period, then you'll never get it .... After that he went nuts and was trying to get his daughter to land a double axel." Leo further explained: "because when you get boobs and hips, the rotation becomes harder and if you don't get that muscle memory before ... I'm not saying it's not possible, but it would be more difficult. That's what everyone thinks." One participant, Ian, described the skating dress as another source of pressure for female skaters: "Sometimes they'll keep their weight down just to fit in their costumes . . . . It's almost like a bride in her wedding dress .... It's such a big part of the way they look in their program and such a big part of their overall package as a skater."

Participants described the distress associated with these body pressures for female skaters. Wes noted: "You can see the stress just kind of when you look at them, and it's almost like a panic at times .... I guess they don't feel good enough and they need to do something to make them better at skating . . . like lose weight or something." These body pressure experiences were also normalized, as Tom suggested: "We joke about it at the rink, but it's almost normal for people to be anorexic or really small because that's sort of like the norm in skating." Tod similarly reported that "sometimes [female skaters] look a little bit anorexic." Participants also discussed a wide range of unhealthy behaviors observed in female skaters due to body pressures, such as "starve," "be hungry when [they] skate," "run for an hour every single day," and "having 
an eating disorder." In stark contrast to the reported eating behaviors of many male skaters ("It's called the seafood diet. You see it, you eat"), Ian noted: "I don't see [female skaters] eat much. A lot of rabbit food." Ron described specific behaviors he has observed in female skaters: "Anything from count calories exactly and only eat certain things to go throw up in the bathroom. Like 'hey, it's weigh-in day, and I just ate so I'm going to go throw up in the bathroom for the next few hours .... Work out more ... skate more." Ron shared a specific example: "There was actually a girl at their rink who starved herself for three days. She was losing her hair and had problems because she just kept wanting to be skinner . . . she looked like a holocaust victim. She just wasn't eating."

In citing these observations, participants vehemently disagreed with the approaches taken by female skaters to change their bodies, as Ike noted:

That's the unfortunate mistake I think a lot of girls make. Especially in this sport. They get it stuck in their head that nothing is $\mathrm{OK}$, even good food. Just like food in general is not OK. So, they get almost to the point of almost starvation in a sense, and that's when your body starts to backfire on you.

Ron reported frustration associated with the body image concerns of female skaters:

I hate it when skinny women call themselves fat. I will yell at them ... . You' re just stupid. Stop talking right now. You have no idea what you're talking about .... Girls will be a twig and be like 'oh my God I'm so fat.' I just want to slap you.

\section{Reasons for the Gendered Body Pressure Experience in Figure Skating}

Participants offered reasons for the differences in body pressure experiences between male and female skaters. These reasons included that female skaters face more extreme body pressures than male skaters who have a physical advantage in the sport. Female skaters were also thought to be more self-critical and male skaters more self-confident.

\section{Female Figure Skaters Face More Extreme Body Pressures.}

Participants suggested while body pressures exist for male skaters, they are more extreme for female peers. Tom noted, "Sometimes we joke about it. But for guys I think it's a lot more laid back than for girls." He further explained: "It doesn't seem like the pressure is the same for guys." Jay reported body ideals are more rigid for women: "People like the females to be one way. You know, there's only one ideal. For men, it's not so much like that." Ron indicated female skaters are weighed more often than their male skating partners: "The girls are [weighed] once a week and the guys are [weighed] every three weeks." Ron attributed differences in body pressures to gender roles: "In pairs especially everyone looks at the girl. No one looks at the guy. We're just kind of there for like the foundation of stuff. We're just showing off the girl, so she's got to look good." Jay reported female skaters face unreasonable demands to change their bodies:

It's more unreasonable with females than with males .... One time last year, one of the girls was told you need to lose 15 pounds just out of the blue ... and you kind of look at her and you think how is she going to lose weight? Like where is it going to come from? ... But for males I think it's a question of more just habits rather than their bodies ... not even in terms of losing weight but maybe you could eat a little bit healthier.

Ike explained why male and female skaters respond to body pressures differently:

The genders handle it differently. So, in my case, the coach tells me, 'I want you to bulk up' or anything like that, I take it as 'ok sounds good.' I have no reservations about it . . . I think that when girls hear they need to lose weight or something like that, there's always a subtle sense of I don't know, there may be a little resistance in a sense because for girls it is more difficult because of their body types compared to guys . . . . Like for guys they're not ashamed that they're not strong enough. You're more... almost excited that you're about to get stronger almost or at least improve. I think that when girls are told they're overweight, it's more an aspect of shame for them.

Participants also reported that unlike female skaters, the culture around male skaters focuses on function over appearance, as Wes described: "As long as [guys are] performing and improving, I don't see much pressure to be starving themselves or anything." Ike described the emphasis on body function in pairs skating: "We're just glorified forklifts in figure skating, right? All we do, we pretty much just lift girls and make them look good. So as long as we have enough strength to do that and then pull off two jumps in a program, then we're good to go pretty much."

At the same time participants acknowledged a differential body pressure experience, some suggested female skaters accept and learn to manage the body pressures associated with elite figure skating, as Ike described:

If it's handled well, it really isn't that much of an issue for anybody as long as the parents are knowledgeable about what's healthy, the coaches are knowledgeable about what is good for their skater, and the girls are understanding that I know I'm going to be pushing my body to what is considered extreme for the average American girl, but at the same time, it's also what is required in this specific sport and they have to understand that if you aren't comfortable pushing your body to the limits of extreme, then maybe figure skating isn't for you.

Leo similarly reported that young skaters need to develop good training habits early and "[Little girls] have to decide if they do want to do it."

Male Figure Skaters Face Fewer Body Pressures Due to Physical Advantages. Other participants noted male skaters have a physical advantage that makes managing body weight, shape, size, and appearance easier. Respectively, Leo and Eli reported, "it's easier for younger guys to stay physically fit automatically" and "typically male skaters don't really have a lot of body fat to start off with." Ron described perceptions of sex differences during puberty: "[Women's] metabolism slows down more than guys . . . so they actually $d o$ have to watch what they eat." Ian highlighted perceived differences in weight gain and fluctuation:

If [men] gain weight, a lot of times they gain weight with muscle, and muscle isn't a bad thing. But with girls, you want them to have that really elegant, long line, that image of a princess .... With guys it's OK to be big sometimes and muscular and frumpy because they're guys. They jump big, they land hard .... I don't know why it started but that's what I 
know right now is that they want the girls to stay a certain weight. Girls also seem to fluctuate a lot more in weight than guys, and it can seem to affect their jumping a lot too.

Ike described a wider margin of acceptable weight loss and weight gain in male skaters due to body type and biomechanics:

If you're going to be a pair girl, more than anyone else, you have a very narrow margin on weight loss or gain that would be healthy. And since they are very lightweight people to begin with in a sense, weight gain or weight loss can be much harder to deal with than for a man who can afford to lose quite a bit of weight and be healthy or put on a bit of weight and be considered healthy also.

Ike further explained:

Guys have a little more room to play with than women because we can afford to have a higher range of body mass percentages or muscle mass distribution because, first of all men tend to jump higher, so that just naturally gives us more air time. So, I think there is generally a little bit less pressure on guys in the sport to really maintain a specific body type. Because in a number of ways basic biomechanics allow us to compensate for what would be a poorer body type than ideal . . . . Girls do definitely feel a bit of pressure to maintain a certain body type. But I wouldn't say it's unjustified at least.

Interestingly, Tom explained a self-selection process may explain the fewer body pressures experienced by male skaters:

If you're doing well, then you most likely already have a good body type for the sport so then there's nothing to even worry about. But obviously there's always a bit more stigma around guy skaters. If it's not going well or you're not a good skater, you're more likely to go and do something else. For girls, it's completely acceptable to be a figure skater so even if you're not as good you'll probably still stay in it.

Female Figure Skaters Are More Self-Critical. Participants suggested that women in general, and female skaters by extension, are more sensitive to body image concerns. Eli stated: "I think girls in general are more prone to that whole like perfect body thing. I don't really think guys, I mean there are some guys that would let it get to them, but I wouldn't say they would care that much." Ben noted that women tend to be more appearance-conscious:

I think for guys they don't really think about their body too much ... . in a sense that I need to lose all this weight like a girl does ... just because girls are a little bit more concerned about the way they look and come off to other people and stuff.

Ian reported women are more self-critical: "[Women] are like 'I look heavy' or 'I feel fat' or 'my legs are getting bigger.' It's just their self-image. They are harder on themselves than other people. They judge themselves."

Participants also reported female skaters tend to address poor performance by criticizing their bodies, as Tod illustrated:

I think that sometimes when girls in our sport think they can't do their thing, they look at their body really negatively. Then they don't treat it as well as they should and get their nutrients. Sometimes they think that if they want to try the hard jumps that the guys are doing, which some of them have very successfully ... they need tinier bodies in order to do it.
Whereas it's actually the opposite. They need to get stronger so they can do it.

Tom similarly noted: "It always seems that boys always jump higher or get bigger jumps and it comes quicker. So maybe for girls it doesn't come as quickly, so they tend to think 'oh I weigh too much.' I don't know. It's just a guess."

Male Figure Skaters Are More Self-Confident. Ian reported men tend to "have a little bit more of an ego sometimes" relative to their bodies: "Some guys definitely are like you're too small, you need to get bigger . . . you don't hear it as much. A lot of guys are really confident . . . like yo, I can do the big tricks. Who cares if I'm overweight or underweight?" Some participants suggested this confidence may aid coping with body pressures in constructive ways. Eli described body pressures as a source of motivation: "I think all the pressures to have the perfect skating body has just like motivated me to keep in shape and not become lazy, eat junk food, or give up the sport. It just motivates me to be closer to that quote 'perfect body."' Sid described body pressures from coaches as a source of constructive feedback:

I just try to take them in not as harshly. They're doing what they think is right to build you as a more adequate and successful skater. But so, I don't necessarily try to cope with it more than I try to run with it, and I don't take it as a put down. I take it as a goal to set and what steps am I going to take to reach this goal?

Sid further explained that body changes must be approached within the limits of reason: "If someone came up and told me something completely unrealistic, I don't think I'd hesitate to tell them, let's step back for a minute and reevaluate what's logistically possible here."

\section{Recommendations for Addressing Body Pressures in Figure Skating}

Given their experiences and observations, participants offered several recommendations for addressing body pressures in figure skating, such as shifting beliefs to promote healthier relationships with body image, eating, and exercise. Sid described the skating culture as one that perpetuates body pressures: "Kids hear they need to cut weight and are aware of this stereotype that figure skaters have eating disorders in order to cut this weight. So, they just sort of assume. It's kind of monkey see, monkey do if you will." Sid suggested debunking the false narratives in skating relative to the body:

I think people need to stop trying to find what is that perfect body for skating because there is no perfect body for skating. Sure, there are things that help make it more appealing and quote, unquote "proper" for the general public, like having long legs, but it's been proven that people can find a way to work around it and create their own style.

Jay recommended being open to different philosophies on skating body ideals: "Open the ideals to not just one body type, but what works best for the skater ... . The emphasis should be on function, not on appearance so much . . . . I'd also get rid of those . . . traditional practices, you know the weigh-ins." Jay further noted: "I mean I think it's just a question of being open minded . . . emphasizing health in general. Not necessarily health because of skating or like related to skating, but just as a human, you know?" 
Other participants discussed the importance of education for all stakeholders, such as discrediting nutrition myths and using qualified professionals to tailor approaches, as Ian noted:

I feel like a lot of coaches try to take control of [weight management] in their skaters. And I feel like if you really want to do that as a skater you should get your advice from a nutritionist who knows exactly what they're talking about. I feel like a lot of people just pull stuff like this is what I did when I was younger so you're going to do that too. That happens so much in skating ... Don't just ballpark that and be like ah this is what my coach said, let's just do that ... go to a professional and get a program from them written exact straight for you.

Tod emphasized coaching education: "All the tests the coaches have to go through to become coaches . . . there should be something about nutrition that educates them." Leo reported coaches need to talk about the body "in a way that's not going to upset the skaters," but questioned "if that's even possible." Ike offered how he would handle these barriers as a coach:

I would say 'I've gathered the profiles of twenty athletes and the average height is this and the average weight is this, the average body size is . . . Those girls made the Olympics so let's try and get you as close as we possibly can to those while still being safe and conscientious about your body type in general" . . . Instead of saying "hey you're overweight, you need to lose weight" .... At least this way you have some sort of actual evidence to back up your request.

Most participants indicated judges play no role, although Sam suggested they offer feedback on skaters' bodies: "[Judges] might say 'I think your body could be . . . if you did this instead, you might not look like . . . like sometimes if you're overweight you might appear like awkward ... you could try a different style and it might help if you lost some weight."' Skaters described how parents' involvement in their child-skaters' nutrition and body image should be positive and limited, as Wes stated: "Maybe not focus on the end result of a change to more of an ideal body but maybe sit down ... and ask [their child-skater] how they feel about it. Because ultimately, it's their body, and if they're happy with it, the parents really shouldn't be trying to change that."

\section{Discussion}

Male skaters were uniquely positioned to observe and discuss the body pressure experiences of female skating peers. Examining these findings through a social constructivist and critical lens yielded insight into the intersection of gender and power, embedded in the sociocultural and historical context of figure skating. Drawing connections between the ideal female skater's body and traditional feminine stereotypes (e.g., Barbie, "a bride in her wedding dress") reflects the hyperfeminization of figure skating over the last century. Since the days of Sonia Henie, figure skating has been a popular form of entertainment showcasing Westernized beauty ideals and princess-like characteristics for women that are perpetually reinforced by the media (Fabos, 2001; Feder, 1994) as well as the structured rules of figure skating. For example, Adams (2011) noted the International Skating Union required skirts for female skaters until 2004. Visual inspection of figure skating costumes demonstrates how women's skating dresses have become increasingly shorter and more revealing over time. These historical accounts help explain the emphasis on appearance for female skaters as described by the male skaters in this study. Further, to "look good" in these costumes, the male skaters suggested female skaters are expected to hide muscularity, but still be strong enough to perform technical requirements. Looking like Barbie but "really strong underneath" attempts to strike a balance between promoting the advancement of technical skills expected by sport spectators while ensuring female skaters still fit easily into traditional gender schema. However, assertions that Barbie's unrealistic proportions would prohibit her even from walking (Norton, Olds, Olive, \& Dank, 1996) demonstrates the contradictory, unrealistic, and unhealthy nature of these ideals for most athletes who have not been genetically endowed with a precise set of physical features.

Reports by male skaters also suggested the female body (e.g., shape, size, weight, appearance, metabolism, fat, menstrual cycles, and perceived physical limitations) is believed to be a primary reason, and target of intervention, for performance problems. Male skaters' descriptions of female skaters going through puberty (e.g., getting "hit really hard") depicts an unwanted, external force disrupting skating careers. The first menstrual cycle was described as a pivotal experience influencing whether female skaters succeeded in figure skating (e.g., "if you don't get your double axel before your first period, then you'll never get it"). These responses represented a disturbing reality that some female skaters are shamed for experiencing a normal and healthy part of becoming a woman. Interestingly, male skaters described men as having certain biological privileges over women that allowed them to excel more easily in figure skating. For example, they explained men have less body fat to start, men gain weight in muscle and women gain weight in fat, women have slower metabolisms and fluctuate more drastically in weight, and men have more natural ability (e.g., to jump higher) that allows them to compensate for a less-than-ideal body. These generalizations, however, are problematic in two major ways.

First, the male skaters in this study had many years of skating experience with competitive success at the elite level. Thus, their perceptions regarding the physical advantages of male skaters were likely limited to observations of a homogenous group of competitive skating peers and excluded many men who did not meet that definition, dropped out, or never attempted figure skating. Second, the perceived physical differences between male and female skaters, though quickly attributed to biological fact, are exaggerated by gendered social processes (Brown, 2006). For example, popular discourse in both society and figure skating that promotes dieting for women (e.g., eat "rabbit food") and fueling for men (e.g., "It's called the seafood diet; you see it, you eat it") serves to weaken female bodies and strengthen male bodies. Thus, the differences observed between male and female skaters' bodies are not merely an inherent biological truth, but exaggerated by differentially reinforced eating practices. Citing McNay (1992), Calogero and Thompson (2010) conjectured, "Part of the problem has been that men's bodies have served as the gold standard for what is good and normal about the human body, and, therefore, by comparison, women's bodies have often been perceived as inferior and deviant from this biological baseline" (p. 169). Although biological differences exist across all people, the gendered hierarchy that emphasizes men as dominating biological prowess and natural skill over women is a socially constructed, ideologically sustained truth that reinforces the gender binary and a patriarchal power structure in figure skating. Notions that female bodies are inherently to blame and can be problematic to skating performance, particularly during and after puberty, helps explain the disproportionate focus on 
reshaping their bodies to fit a specific mold; an emphasis especially evident in the pairs discipline.

Although body pressures faced by female skaters were acknowledged as more intense and extreme, the narratives shared by male skaters suggested female skaters are also held personally responsible for being affected by them (e.g., "they judge themselves more than other people"). Further, despite that female skaters are perceived to be vulnerable, they are still subjected to body criticism and shaming. A blame-the-victim narrative is reflected in the general eating literature where eating disorders are falsely believed to be a choice (Bulik, 2016). Busanich and McGannon (2010) discussed the importance of diverging from traditional conceptualizations of body image concerns and disordered eating as individual deficits and critically evaluating social, cultural, and historical influences, rife with intersections of gender and power, that inform the development and maintenance of eating pathology. These authors pose a critical question: "Who gains power by supporting the conceptualization of female athletes' bodily experiences as pathological and who is disempowered?" (p. 395). In this examination, attributing body image concerns and disordered eating behaviors to personal faults disempowers female skaters who experience them.

Further, the language and tone with which a few male skaters described their reactions to the body pressure experiences of female skaters was emphatic and at times verged on condescending, reflective of an underlying power structure where men are deemed acting authorities who know best (e.g., "you might want to watch what you eat") and women know little (e.g., they make "unfortunate mistakes," are "just stupid," and "have no idea what [they're] talking about"). Foucault (1977) argued every society has a "regime of truth" (p. 131) governing how and by whom truth is deciphered. In this study, a few male skaters appeared to perceive themselves as more expert in the body pressure experiences of female skaters than female skaters themselves. Further, the apathetic approach male skaters took to describing highly pathological and dangerous eating and exercise behaviors of their female skating peers was particularly troublesome and a reflection of the broader figure skating culture through which they have been socialized (e.g., "I don't know why it started but that's what I know right now is that they want the girls to stay a certain weight"). It is not illogical to anticipate some of these male skaters will eventually become skating coaches; thus, the broader sociocultural skating culture informing their attitudes toward eating pathology are vitally important. There have been various calls to action in the popular media regarding unhealthy eating and exercise behaviors observed in figure skaters, such as Ryan's (1995) book Little Girls in Pretty Boxes and former figure skaters sharing their struggles with an eating disorder (e.g., Kirk, 2009). However, the findings of the present study suggest the unsafe, and even fatal, consequences of disordered eating and exercise are not fully appreciated across all levels of figure skating and remain a normalized part of some skating cultures (e.g., "We joke about it at the rink, but it's almost normal for people to be anorexic"). These perceived truths, including blame-the-victim narratives and normalizations of eating pathology, diminish the sense of urgency with which stakeholders should promote the health, wellness, and performance of all skaters.

Importantly, the participants made recommendations to reduce body pressures in figure skating, such as changing belief systems, debunking myths, seeking professional expertise, and getting educated. It was encouraging that many of these recommendations represented sound resolutions, which perhaps represented exposure to some recent trends in nationwide media campaigns that promoted more progressive views and body diversity for women. Other recommendations reflected a potentially unhealthy focus on weight and fat, such as having coaches use body fat percentages of elite skaters as sources of comparison. Further, some modes of offering support were not entirely productive (e.g., telling them to stop or conform to body ideal expectations). Some participants suggested coaches should be challenged if goals are unrealistic and seek alternative professional expertise. Yet, the safety and space to voice these concerns and self-advocate is a luxury for the empowered. For example, male skaters suggested they are not affected by body pressures to the same extent because the pressures they face are less intense and their personal characteristics offer immunity (e.g., more confidence, better ability to master bigger, higher jumps more quickly, and don't "let it get to them"). An added layer of complexity was represented by male skaters' reports of parents and coaches using approaches that outwardly criticized or blamed the person when addressing female skaters (e.g., being fat), but targeted behaviors when addressing male skaters (e.g., make healthier choices). In contrast, the results of this study suggest the pressure experienced by female skaters can greatly compromise their ability to speak up, advocate for their body, and challenge weight recommendations from coaches, who inherently have more power than athletes. The male skaters, although generally opposed to the unhealthy eating and exercise behaviors observed in female skaters, were less attune to how they could serve as allies-a change that would require the support of coaches, parents, judges, governing body representatives, and other stakeholders who shape the figure skating culture.

Together, our results shed light on how both observed and perceived differences between male and female skaters are socially constructed into an ideal masculinity that is highly privileged and praised over an ideal femininity that is minimized in comparison. Notions that male skaters have biological advantages (e.g., lower body fat), athletic prowess (e.g., jumping more easily), and psychological superiority (e.g., more confident, better coping skills) over female skaters may explain the disproportionate focus on 'fixing' the bodies of female skaters and the more extreme pressures they experience to change themselves. Reports that a larger range of body ideals are acceptable for male skaters, in comparison to female skaters who face a narrow and often impossible body standard, further demonstrates a hegemonic power structure that allows men to more readily achieve body ideals and be more dominant in figure skating. The male skaters in this study seemed to, unknowingly, enforce this ideologically-sustained hierarchy to protect an ideal masculinity, reflecting the broader historical and sociocultural context in which they train, compete, and socialize.

In its earliest iteration, figure skating was an art form pursued by men for intrinsic reasons versus competition; the demonstration of athletic skills that increased the risk of falling, was thought to interfere with the grace of figure skating (Adams, 2011). Despite its origins as an expressive form of masculinity in 18th century England (Adams, 2007), male skaters are seldom viewed as manly in contemporary figure skating. As figure skating has transformed into a hyperfeminized activity, men who participate risk their gender, sexual identity, and social status being called into question. Anxieties over perceptions of effeminacy have shaped media constructions of exaggerated masculinity and informed rules designed to protect masculine portrayals of male skaters. Masculinizing male skaters helps figure skating be seen as a true sport and male figure skaters be perceived as athletes, versus artists, to promote viewership and credibility among fans who expect 
exhibitions of male strength and power, as in American football (Adams, 2011). Male skaters have thus been encouraged to prove their masculinity by vetting behavior, movement, costume, music, and appearance for symbols of effeminacy. This historical context offers critical insight into how and why male skaters in this study responded in ways that appeared to similarly protect masculinity.

For example, because male athletes are not supposed to be concerned with appearance explains their emphasis on the functional aspects of skating and why the interviews so readily shifted to the appearance concerns of female skaters instead. Further, the pattern of responses by male skaters emphasized differences between female skaters and themselves relative to the importance of costume, skill level, physique, biology, personal characteristics, and perceived levels of vulnerability to disordered eating and exercise behaviors. Given that jumping is considered the "hard stuff" because of its association with a type of strength and athleticism more stereotypically associated with masculinity (Adams, 2011, p. 196), it is not surprising male skaters emphasized the importance of, and superior ability to perform, this specific skill. Perhaps these symbols of binary gender distinction were even better exemplified in descriptions of the pairs discipline. Striving to achieve an "80-pound weight difference" between pairs partners, although claimed a requirement for performing difficult skills safely, also serve to exaggerate the appearance of difference between male and female skaters. Male skaters also explained role differentiation in pairs skating whereby men are "glorified forklifts" while women are "shown off" for decorative purposes. In these ways, male skaters distance themselves from femininity by looking and behaving differently in a context where their masculinity may be questioned. However, reinforcing this heteronormative image hurts male and female skaters. Notions that female skaters are to be displayed for their beauty minimizes their contributions and reinforces objectification of the female body. At the same time, reducing the role of male skaters to a prop squanders their freedom to perform in ways that express a once highly regarded art form for men. In this study, male skaters focused almost exclusively on jumping and lifting partners when describing their abilities, as if these skills were their single biggest contribution; however, male skaters can and do offer much more to figure skating that should be freely recognized.

Importantly, figure skating is rife with opportunity for positive, safe, and equitable experiences that promote benefit and reduce risk for skating participants, particularly with regard to building and sustaining healthy relationships with their bodies. Awareness is a key impetus for change. Moving forward, skaters and other stakeholders including governing body representatives, coaches, judges, and parents could position themselves as change agents of problematic norms with greater knowledge of, not just nutrition, but the historical, sociocultural, and gendered context that has shaped the dominant discourse in some figure skating environments. To inform future research, the limitations of this study must also be considered. The perspectives shared were limited to 13 male figure skaters competing at an elite level in singles and pairs disciplines. Elite participation implies greater pressure to perform, which likely contrasts the experience of skaters at lower competitive or recreational levels. In addition to examining other skating levels and disciplines, it is critical to examine body pressures beyond the traditional gender binary (male, female), understand the experiences of skaters across gender and sexual identities and consider the intersection of multiple identities that also include race, ethnicity, and social class. Finally, the results of this study may serve as a springboard to investigate body pressures, body image concerns, and unhealthy eating and exercise behaviors in other sports from sociocultural, historical, and critical perspectives.

\section{References}

Adams, M.L. (2007). The manly history of a 'girls' sport': Gender, class and the development of nineteenth-century figure skating. The International Journal of the History of Sport, 24(7), 872-893. doi:10. 1080/09523360701311752

Adams, M.L. (2010). From mixed-sex sport to sport for girls: The feminization of figure skating. Sport in History, 30(2), 218-241. doi:10.1080/17460263.2010.481208

Adams, M.L. (2011). Artistic impressions: Figure skating, masculinity, and limits of sport. Toronto, Canada: University of Toronto Press.

Braun, V., \& Clarke, W. (2006). Using thematic analysis in psychology. Qualitative Research in Psychology, 3, 77-101. doi:10.1191/ 1478088706qp063oa

Brown, D. (2006). Pierre Bourdieu's "Masculine Domination" thesis and the gendered body in sport and physical culture. Sociology of Sport Journal, 23(2), 162-188. doi:10.1123/ssj.23.2.162

Bulik, C.M. (2016). Towards a science of eating disorders: Replacing myths with realities: The fourth Birgit Olsson lecture. Nordic Journal of Psychiatry, 70(3), 224-230. PubMed ID: 26355341 doi:10.3109/ 08039488.2015.1074284

Busanich, R., \& McGannon, K.R. (2010). Deconstructing disordered eating: A feminist psychological approach to the body, food, and exercise relationship in female athletes. Quest, 62(4), 385-405. doi:10.1080/00336297.2010.10483656

Calogero, R.M., \& Thompson, J.K. (2010). Gender and body image. In J.C. Chrisler \& D.M. McCreary (Eds.), Handbook of gender research in psychology (pp. 153-184). New York, NY: Springer.

Cummins, L.F. (2007). Figure skating: A different kind of youth sport. Journal of Clinical Sport Psychology, 1, 390-401. doi:10.1123/jcsp. 1.4 .390

Denzin, N.K. (2017). Critical qualitative inquiry. Qualitative Inquiry, 23, 8-16. doi:10.1177/1077800416681864

Fabos, B. (2001). Forcing the fairytale: Narrative strategies in figure skating competition coverage. Culture, Sport, Society, 4(2), 185-212. doi:10.1080/713999817

Feder, A.M. (1994). "A radiant smile from the lovely lady" overdetermined femininity in "ladies" figure skating. Drama Review, 38(1), 62-78. doi:10.2307/1146356

Foucault, M. (1977). Truth and power. In C. Gordon (Ed.), Power/ knowledge: Selected interviews and other writings 1972-1977 by Michael Foucault (pp. 109-133). New York, NY: Pantheon Books.

Foucault, M. (1988). The ethic of care for the self as a practice of freedom. In J. Bernauer \& D. Rasmussen (Eds.), The final Foucault (pp. 1-20). Cambridge, MA: MIT Press.

Galli, N., \& Reel, J.J. (2009). Adonis or hephaestus? Exploring body image in male athletes. Psychology of Men \& Masculinity, 10(2), 95108. doi:10.1037/a0014005

Kirk, J. (Producer). (2009, February 22). Skating's not-so-secret shame. Skating the Issue. [Web log message] Retrieved from http://trueslant .com/jenniferkirk/2009/07/05/skatings-not-so-secret-shame/

Lee, J. (1994). Menarche and the (hetero) sexualization of the female body. Gender and Society, 8(3), 343-362. doi:10.1177/089124394008003004

McGarry, K. (2005). Mass media and gender identity in high performance Canadian figure skating. The Sport Journal, 8(1). Retrieved from https://thesportjournal.org/article/mass-media-and-gender-identity-inhigh-performance-canadian-figure-skating/ 
McNay, L. (1992). Foucault and feminism: Power, gender, and the self. Cambridge: Polity Press.

Norton, K.I., Olds, T.S., Olive, S., \& Dank, S. (1996). Ken and Barbie at life size. Sex Roles, 34(3-4), 287-294. doi:10.1007/ BF01544300

Ryan, J. (1995). The making and breaking of elite gymnasts and figure skaters-Little girls in pretty boxes. New York: Warner Books.

Smith, B., \& McGannon, K.R. (2018). Developing rigor in qualitative research: Problems and opportunities within sport and exercise psychology. International Review of Sport and Exercise Psychology, 11(1), 101-121. doi:10.1080/1750984X.2017.1317357

Story, C., \& Markula, P. (2017). 'I had one year in junior and then I was too old:' Structural age ryles and the girlification in Canadian elite women's figure skating. Sport in Society, 20(9), 1223-1240. doi:10.1080/17430437.2016.1269086
Tracy, S.J. (2010). Qualitative quality: Eight 'big-tent' criteria for excellent qualitative research. Qualitative Inquiry, 16, 837-851. doi:10. 1177/1077800410383121

Voelker, D.K., Petrie, T.A., Reel, J.J., \& Gould, D. (2018). Frequency and psychosocial correlates of eating disorder symptomatology in male figure skaters. Journal of Applied Sport Psychology, 30(1), 119-126. doi:10.1080/10413200.2017.1325416

Voelker, D.K., \& Reel, J.J. (2015). An inductive thematic analysis of competitive female figure skaters' experiences of weight pressure in sport. Journal of Clinical Sport Psychology, 9, 297-316. doi:10. 1123/jcsp.2015-0012

Voelker, D.K., \& Reel, J.J. (2018). An inductive thematic analysis of male competitive figure skaters' experiences of weight pressure in sport. Journal of Clinical Sport Psychology, 9(4), 297-316. doi:10.1123/ jcsp.2015-0012 